\title{
Directly observable G band splitting in Raman spectra from individual tubular graphite cones
}

\author{
N. G. Shang ${ }^{\text {a, * }, \text { S. R. P. Silva }}{ }^{\text {a }}$, X. Jiang ${ }^{\text {b }}$, P. Papakonstantinou ${ }^{\text {c }}$ \\ ${ }^{a}$ Advanced Technology Institute, University of Surrey, Guildford, Surrey, GU2 7XH, \\ United Kingdom \\ ${ }^{\mathrm{b}}$ Institute of Materials Engineering, University of Siegen, Paul-Bonatz-Strasse 9-11, \\ 57076, Siegen, Germany \\ ${ }^{c}$ Nanotechnology and Integrated Bio-Engineering Centre, University of Ulster, Shore \\ road, Newtownabbey, BT37 0QB, United Kingdom
}

\begin{abstract}
Individual tubular graphite cones (TGCs) are characterized using Raman spectroscopy at room temperature. A split $\mathrm{G}$ band at $1569-1587 \mathrm{~cm}^{-1}$ is directly observed from the root to the tip of TGCs without requiring surface electromagnetic and chemical enhancement effects. The $\mathrm{G}$ band can be deconvoluted into two Lorentzian peaks at $1571 \pm 2$ and 1584 $\pm 3 \mathrm{~cm}^{-1}$, which may be attributed to the resonance enhancement of a single chirality excitation between the innermost tubes and other constituent tubes of the TGCs, respectively. Results suggest that the splitting of $\mathrm{G}$ band can be envisaged as a fingerprint for monochirality of tubular carbon nanomaterials.
\end{abstract}

*Corresponding author. E-mail address: ngshang@ hotmail.com (N. G. Shang) 


\section{Introduction}

Raman spectroscopy is one of the most powerful tools developed for the structural characterization of carbon based materials, such as diamond, carbon nanotube (CNT), fullerene, graphene and graphite etc [1-8]. In particular, it can distinguish the type (multiwall, metal or semiconducting single-wall) of CNTs based on the low-frequency radial breathing modes (RBMs) and the unique profile or line-shape of split G bands. However, it is quite difficult to determine multiwall carbon nanotubes (MWCNTs) in the presence of graphite due to the lack of the specific RBMs and branched G bands in the Raman spectra. The main reason is either higher density of structural defects or harmful local environments around MWCNTs make the RBM silent or the G band degenerate [9]. To date, the multiple split G band in MWCNTs was only observed with a surface enhanced Raman technique on silver substrates [10]. Recently, we reported the growth of monochiral tubular graphite cones (TGCs), whose structure is similar to MWCNTs, on hundred micrometer sized carbon spherules $[11,12]$. They not only are highly crystalline with a single-crystal nanotip in nature, but also have long distance from the Si substrate, enabling us to probe individual TGCs without the influence of the substrate and other extraneous factors such as strain, bundling and deformation etc [13, 14]. TGCs have also been reported with preferential zigzag monochirality detected by an electron diffraction technique in our previous work $[11,15]$, offering a stable platform to study monochirality behaviour of tubular carbon based materials utilizing conventional Raman spectroscopy. This is more convenient than electron diffraction techniques currently used $[11,15]$. In this paper, we systematically investigate Raman spectra from different regions along asgrown individual TGCs at room temperature. The relationship between the splitting of G 
band and the crystalline quality of tubular carbon nanomaterials is discussed. In order to assign the Raman mode symmetry, polarized data for the G band feature of TGCs are systematically studied.
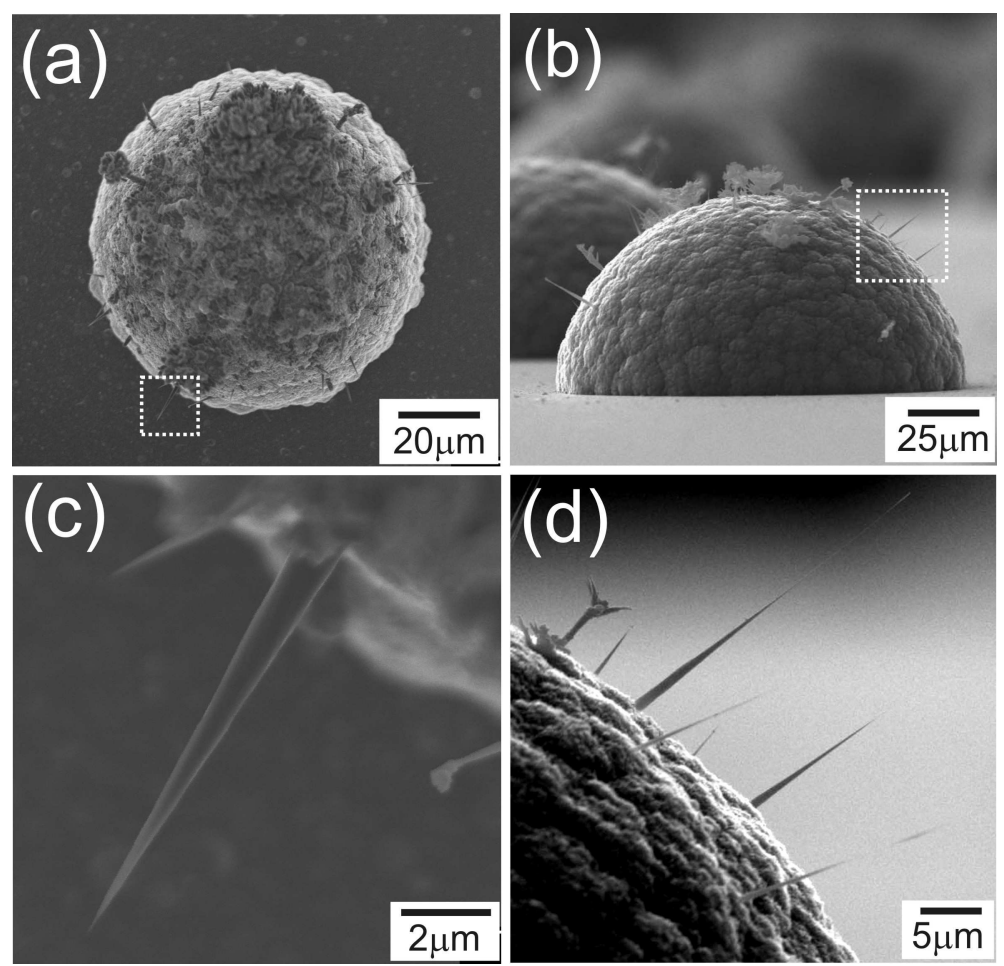

Figure 1. Top view (a) and cross-sectional (b) SEM images of the TGC sample; (c) and (d) are enlarged images of the marked area in (a) and (b).

\section{Experimental details}

TGCs were grown by a microwave assisted plasma chemical vapour deposition using insitu-evaporated Fe catalysts [12]. As-grown TGCs were found to separately stand on the top of perfect carbon semi-spherules with a diameter of $85-150 \mu \mathrm{m}$ as shown in Figure 1. The TGCs are about $4-20 \mu \mathrm{m}$ long and have a root diameter of $0.3-1 \mu \mathrm{m}$. Some TGCs have a straight, thin carbon nanotube tip of $10-20 \mathrm{~nm}$ diameter. The majority of 
TGCs have a spatial separation in the range of $2-5 \mu \mathrm{m}$ or more, making it possible to investigate their individual structure by Raman scattering. Raman scattering was carried out at room temperature using a $514 \mathrm{~nm} \mathrm{Ar}^{+}$laser in a Renishaw spectrometer with a spectral resolution of $2 \mathrm{~cm}^{-1}$ and a spatial resolution better than $1 \mu \mathrm{m}$. The polarization measurement was carried out by continuously rotating the TGC to change the angle ( $\theta)$ between the axis of an individual TGC and the polarization direction of the incident laser with a VV configuration. The incoming and scattered polarised light propagates parallel, but perpendicular to the axis of TGCs. Each step of the sample rotation is performed with care to ensure the laser spot focuses on almost same position of the TGC with the carbon semi-spherule as a reference. The angles were calculated from recorded optical CCD images with an accuracy of $\pm 3^{\circ}$.

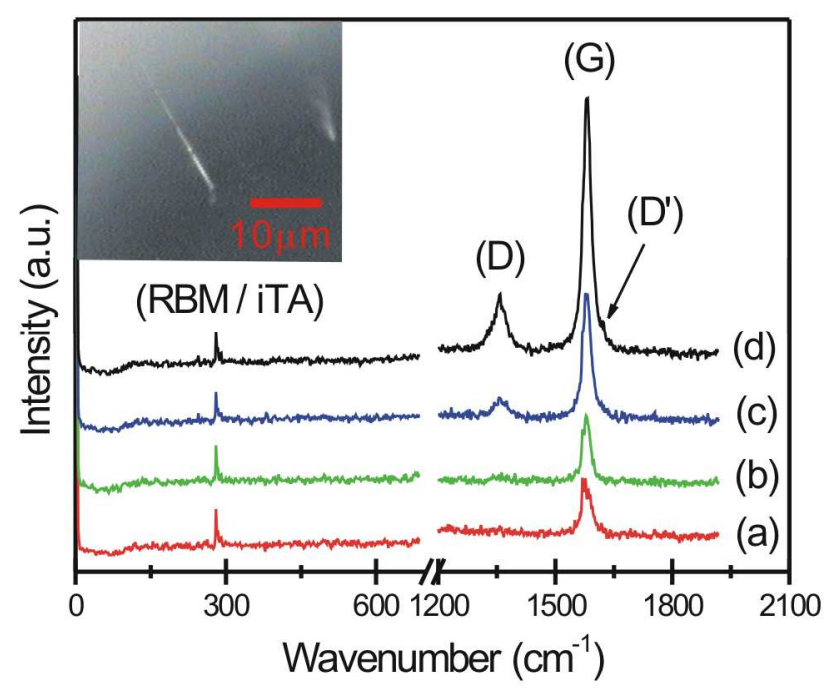

Figure 2. Raman spectra taken from different regions of an individual $17 \mu \mathrm{m}$ long TGC: at about 15 (a); 9 (b); 6 (c) and $3 \mu \mathrm{m}$ (d) away from the root. The inset is a typical optical micrograph of individual TGCs. 


\section{Results and discussion}

Figure 2 shows a series of Raman spectra taken from different regions of a $17 \mu \mathrm{m}$ long TGC. The data at $12 \mu \mathrm{m}$ was corrupted and not usable. The inset is a typical optical micrograph of individual TGCs. It can be observed that there are two regions of peaks: a low-frequency RBM mode at $280 \mathrm{~cm}^{-1}$ and a high-frequency $\mathrm{G}$ band at around $1582 \pm 2$ $\mathrm{cm}^{-1}$, throughout the whole TGC. Another two defect-related peaks: a D band at $1359 \mathrm{~cm}^{-}$

${ }^{1}$ and a D' band at $1622 \mathrm{~cm}^{-1}$, were found close to the root region of TGCs (curves $\mathrm{c}$ and $\mathrm{d}$ of Figure 2). The low-frequency peak has similar intensities and a full width at half maximum (FWHM) of approximately $3 \mathrm{~cm}^{-1}$, while the $\mathrm{G}$ band exhibits decreasing intensities and a FWHM of about $25-35 \mathrm{~cm}^{-1}$ from the root to the tip of TGCs.

As discussed in the literature for SWCNTs and MWCNTs with the innermost nanotube diameter of less than $2 \mathrm{~nm}$, the low-frequency mode observed in this study is believed to originate from the RBM of the thinner innermost carbon tube of TGC, which has been shown to have a diameter of around $2 \mathrm{~nm}$ [11]. However, the variation in peak intensity is quite small in different regions of TGCs and no enhanced resonant, stronger peak was found at the tip. Therefore, the low-frequency mode could also be assigned as an in-plane transverse acoustic (iTA) phonon branch mode of graphite, which is located at $300 \mathrm{~cm}^{-1}$ in theory and at $288 \mathrm{~cm}^{-1}$ in experiment for highly crystalline graphite whiskers $[16,17]$. As for the $\mathrm{G}$ bands, they appear to be branched with either a shoulder or being made of two sub-peaks. Figure $3(b-d)$ shows the enlarged regions of $G$ band taken from the root 

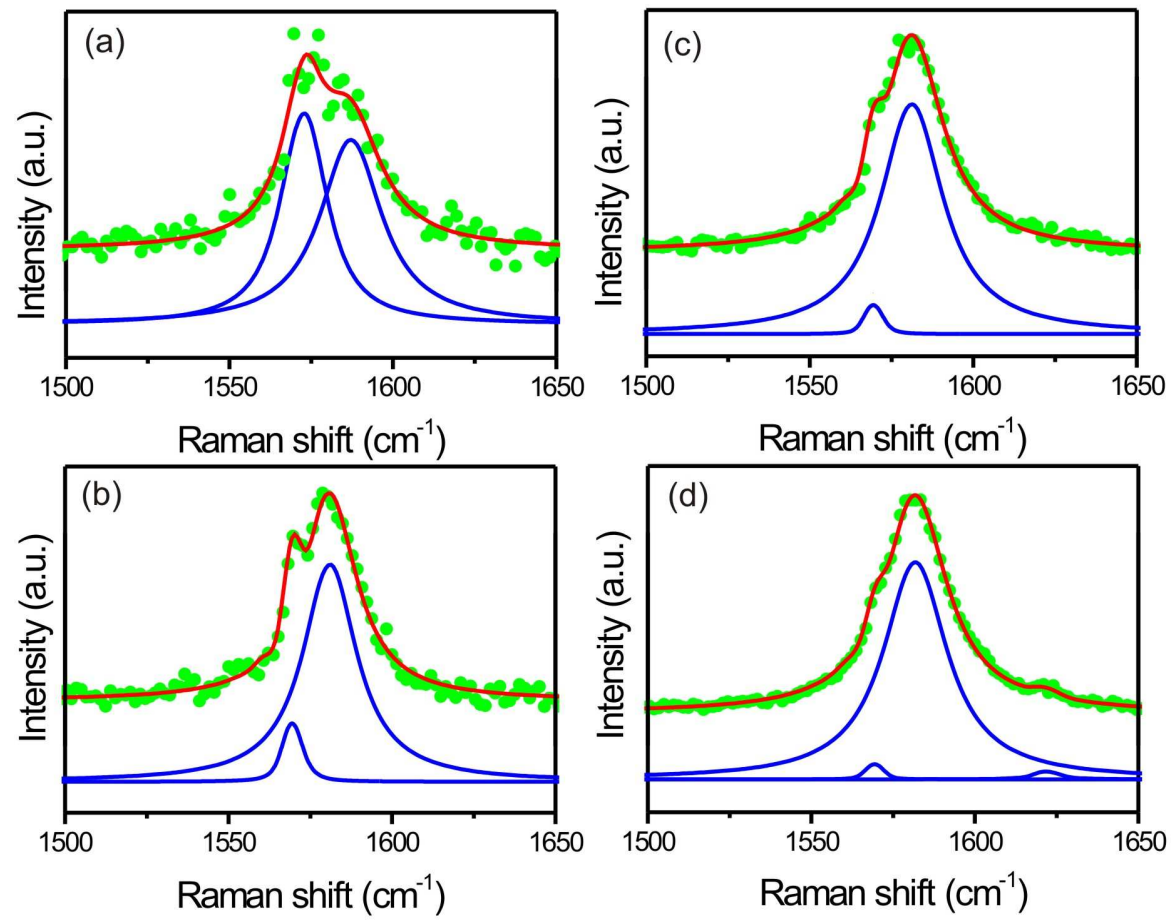

Figure 3. $(a-d)$ Lorentzian line-shape fits of the $G$ band in the corresponding Raman spectra $(a-d)$ in Fig. 2. The blue curves are the deconvoluted peak fits and the red ones are the total peak fits.

and middle regions of TGCs. It is clear that all spectra can be fitted by two Lorentzian sub-peaks with frequencies of $1569-1570\left(\omega_{\mathrm{G}}^{-}\right.$, sub-peak $\left.\mathrm{A}\right)$ and $1581-1582 \mathrm{~cm}^{-1}\left(\omega_{\mathrm{G}}^{+}\right.$, sub-peak B). The FWHMs of sub-peaks A and B are about $3-4$ and $20-24 \mathrm{~cm}^{-1}$, respectively. The integrated intensities of sub-peak B are much larger than those of subpeak A, with the ratio range of $13-85$. However, at the measurement spot very close to the tip (Figure 3a), the Raman spectrum can be deconvoluted into two well defined Lorentzian sub-peaks with the frequencies of 1573 and $1587 \mathrm{~cm}^{-1}$. Except for the slight up-shift $\left(4-6 \mathrm{~cm}^{-1}\right)$ in wavenumber possibly due to the phonon dispersion induced by 
circumferential quantum confinement in the tube compared to other regions of the TGC [18], the FWHM of sub-peak A at $1573 \mathrm{~cm}^{-1}$ is quite large up to $15 \mathrm{~cm}^{-1}$ and the two subpeaks have comparable integrated intensities with a ratio as small as 1.2. All data fits (peak position, FWHM, integrated intensity and ratio) of the two sub-peaks for the $G$ band from the root to the tip of individual TGCs are shown in Table 1. Figure 4 shows the integrated intensity of two sub-peaks as a function of the distance measured from the root to the tip of an individual TGC. From the root to the tip of TGCs, the integrated intensity of sub-peak A and their corresponding FWHM gradually increase, while the integrated intensity of sub-peak B and the integrated intensity ratio of two sub-peaks greatly decrease and their FWHM remains almost constant.

Table 1. All data fits (position, FWHM, integrated intensity and ratio) of two sub-peaks for the $\mathrm{G}$ band from the root to the tip of individual TGCs.

\begin{tabular}{|c|c|c|c|c|c|c|c|}
\hline \multirow{2}{*}{ Distance } & \multicolumn{3}{|c|}{$\omega_{\mathbf{G}}^{-}$Sub-peak (A) } & \multicolumn{3}{|c|}{$\omega^{+}$Sub-peak (B) } & \\
\cline { 2 - 7 } & $\begin{array}{c}\text { Position } \\
\left(\mathrm{cm}^{-1}\right)\end{array}$ & $\begin{array}{c}F W H M \\
\left(\mathrm{~cm}^{-1}\right)\end{array}$ & $\begin{array}{c}\text { Integrated } \\
\text { intensity }\left(I_{A}\right)\end{array}$ & $\begin{array}{c}\text { Position } \\
\left(\mathrm{cm}^{-1}\right)\end{array}$ & $\begin{array}{c}F W H M \\
\left(\mathrm{~cm}^{-1}\right)\end{array}$ & $\begin{array}{c}\text { Integrated } \\
\text { intensity }\left(I_{B}\right)\end{array}$ & $\mathbf{I}_{\mathbf{B}} / \mathbf{I}_{\mathbf{A}}$ \\
\hline $\mathbf{3}$ & 1569.2 & 2.8 & 543.2 & 1581.8 & 23.6 & 46171.8 & 85.0 \\
\hline $\mathbf{6}$ & 1569.8 & 3.0 & 556.8 & 1581.2 & 23.2 & 22490.2 & 40.4 \\
\hline $\mathbf{9}$ & 1569.7 & 4.1 & 759.9 & 1580.9 & 20.0 & 9789.3 & 12.9 \\
\hline $\mathbf{1 5}$ & 1572.8 & 15.2 & 4413.0 & 1587.1 & 23.0 & 5357.3 & 1.2 \\
\hline
\end{tabular}




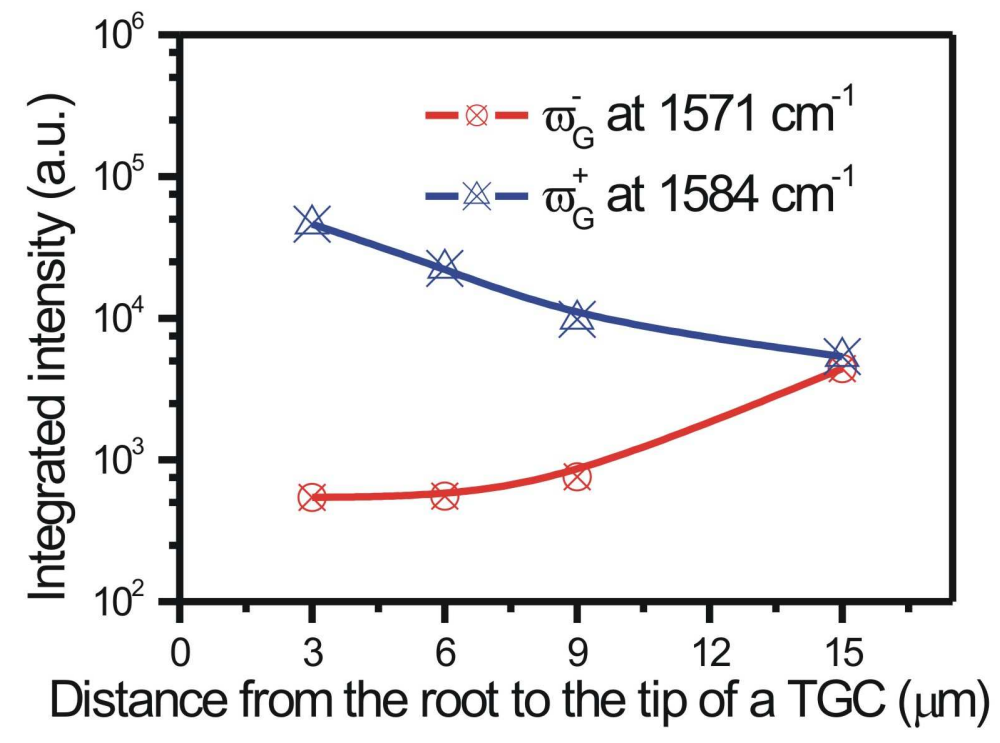

Figure 4. Integrated intensity of low-frequency $\left(\omega_{\mathrm{G}}^{-}\right)$and high-frequency $\left(\omega_{\mathrm{G}}^{+}\right)$sub-peaks as a function of the distance measured from the root to the tip of an individual TGC.

Contrary to the RBM, the G band in SWCNTs shows a more complex spectral feature due to the curvature induced symmetry-breaking effect in the Brillouin zone of SWCNTs. Both theoretical calculation and experimental measurements show that the $\mathrm{G}$ band of SWCNTs consists of six Raman-active modes with the symmetry of $A\left(A_{1 g}\right), E_{1}\left(E_{1 g}\right)$ and $\mathrm{E}_{2}\left(\mathrm{E}_{2 \mathrm{~g}}\right)[18,19]$. All of these are not always visible or distinguishable, and strongly dependant on the different growth methods used to prepare samples. Usually, in a majority of samples two featured peaks appear: a higher-frequency $\left(\omega^{+}{ }_{G}\right)$ sub-peak at about $1591 \mathrm{~cm}^{-1}$ and a lower-frequency $\left(\omega_{\mathrm{G}}^{-}\right)$sub-peak in the range of $1500-1587 \mathrm{~cm}^{-1}$, 
associated with atomic vibrations along the axis and circumference of CNTs, respectively. Semiconducting and metallic nanotubes are found to have a similar Lorentzian line-shape and an identically narrow FWHM line-width of $6-15 \mathrm{~cm}^{-1}$ for the $\omega^{+}{ }_{\mathrm{G}}$ peak. A remarkably different line-shape and a dissimilar FWHM line-width are observed for the $\omega_{\mathrm{G}}^{-}$peak, i.e., a Lorentzian line-shape with narrow FWHM line-width for semiconducting SWCNTs and a Breit-Wigner-Fano line-shape with broad FWHM line-width for metallic SWCNTs, due to different surface plasmon coupling [18, 19]. The present $G$ band observed from the root to the tip of TGCs is also made of a high-frequency sub-peak and a low-frequency sub-peak. Two sub-peaks may have identical origins as that of SWCNTs, i.e. atomic vibrations along the axis and circumference of CNTs, respectively. The lowfrequency sub-peak located at $1569-1573 \mathrm{~cm}^{-1}$ with a narrow FWHM of $3-15 \mathrm{~cm}^{-1}$, is in agreement with those observed for SWCNTs. What is the most interesting is that the integrated intensity of the low-frequency sub-peak increases from the root to the tip of TGCs, with decreasing of carbon nanotube walls involved. This phenomenon appears to be counter-intuitive, but demonstrates that the low-frequency sub-peak originates from atomic vibrations along the circumference of the innermost single tube rather than other outer layer tubes. In this case, there could be a singularity or an enhancement of resonance at the tip apex itself for a monolayer graphene of the single tube involved. The innermost nanotube of the TGC could be semiconducting based on the Lorentzian lineshape of the low-frequency sub-peak. The high-frequency sub-peaks from the root and middle regions of TGCs are mainly at about $1582 \mathrm{~cm}^{-1}$ with broad FWHM of $20-24 \mathrm{~cm}^{-}$ ${ }^{1}$, remarkably different from those (1591 and $6-15 \mathrm{~cm}^{-1}$ ) of SWCNTs. As a typical graphite peak, the peak at about $1582 \mathrm{~cm}^{-1}$ with a FWHM of more than $14 \mathrm{~cm}^{-1}$ is not 
only observed in all MWCNTs but also appears in SWCNT bundles and some isolated metallic SWCNTs [3]. Another critical feature of the present spectra obtained is that in contrast with the low-frequency sub-peak from the innermost tube, the integrated intensity of the high-frequency sub-peak greatly decreases from the root to the tip of TGCs (Figure 4), having a trend similar as to the number of constituent graphene sheets in a TGC. This strongly collaborates that the high-frequency sub-peak should be ascribed to collective atomic vibrations along the axis of constituent all carbon tubes rather than the innermost individual tube of TGCs only, because all carbon atoms on the wall contribute to Raman scattering.

Figure 5a shows a typical polarized $\mathrm{G}$ band taken from a position near the tip of an individual TGC. It can be deconvoluted into two Lorentzian peaks at 1570 and $1582 \mathrm{~cm}^{-1}$, respectively. With the change of angle $(\theta)$ between the axis of the TGC and the polarization of light from 0 to $90^{\circ}$, the integrated intensity of the two sub-peaks and their sum are almost constant (Fig.5b). The slight variation in the intensities may be due to the system and measurement errors, but at $20^{\circ}$ there is a slight increase in the $\omega_{\mathrm{G}}^{-}$peak which we attribute to resonance in the innermost nanotube $\mathrm{G}$ mode. Obviously, these TGCs are different from SWCNTs and aligned MWCNTs [20, 21], in which both show strong angular dependence of the polarized $\mathrm{G}$ band with the highest intensity occurring when the incident radiation is polarized parallel to the tube axis. However, some discrepancies with 

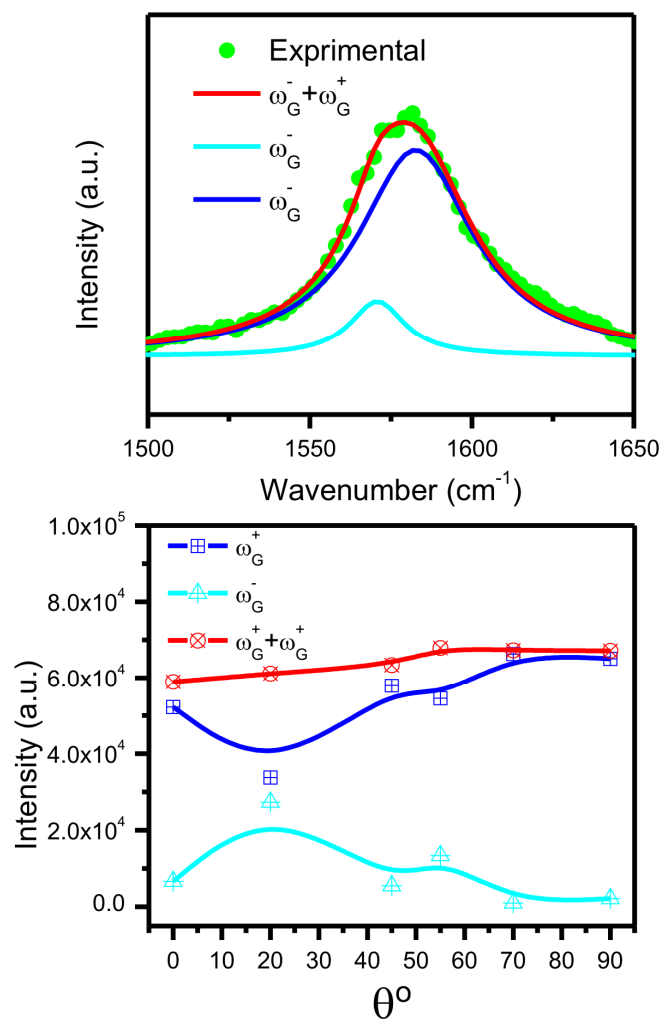

Figure 5. (a) A typical polarized $G$ band; (b) Integrated intensity of $\omega^{+}{ }_{G}, \omega_{G}^{-}$and their sum as a function of the angle between the axis of TGCs and the polarization of laser where the data is collected approximately $12 \mu \mathrm{m}$ from the base of a $18 \mu \mathrm{m}$ long TGC.

theoretic predictions and unusual $\mathrm{G}$ band intensity behaviour are found from individual SWCNT of even the simplest tube system due to depolarization effects or complex multipolar electronic resonance antenna effects [22]. Here, when compared to a SWCNT, the single TGC is a complex integrated system of co-axial tubes and the depolarization effects should be relaxed like in MWCNTs [21], leading to optical response unchanged for the polarized light. This can't be simply ascribed to the diameter variation of the onedimensional structural TGC [23]. For the simple SWCNTs, the difference of electronic 
structure makes optical antenna effect stronger for metallic than for semiconducting nanotubes [24]. For the TGCs, its structural monochirality with special electronic structures may breakdown the symmetry of structure and induce polarization-independent optical resonant absorption to occur for the intersubband transition [25, 26], completely smearing the optical antenna effect, in which the optical absorption takes place only when the light polarization is parallel to the nanotube axis. In this case, the angular dependences measured do not reflect the mode symmetries and are not compatible with the theoretic predictions for symmetry.

It is interesting to explore reasons as to why we can directly observe both the lowfrequency mode and split G mode in individual TGC without the help of any auxiliary techniques. Jantoljak et al. reported the low-energy Raman-active phonons from MWCNTs purified by a liquid-phase separation method [27]. Benoit et al. only observed the low-frequency Raman peak from the purified MWCNTs by the oxidation purification procedure [28]. Zhao et al. found the RBMs from the purified MWCNTs after removing the co-deposited carbon nanoparticles by infrared irradiation in air at $500{ }^{\circ} \mathrm{C}$ for $30 \mathrm{~min}$ [29]. From the same purified samples, he reported multiple split $G$ bands in the MWCNTs by using a surface enhanced Raman technique [10]. Thus, the high-purity or highly crystalline quality of material is a prerequisite for directly detecting both lowfrequency mode and split $\mathrm{G}$ bands. This is considered suitable in high-quality materials, which show various non-resonant phonon scattering to be minimal. In our studies, asdeposited TGCs are of high crystalline quality, as evidenced by the very weak defectinduced $\mathrm{D}$ and $\mathrm{D}^{\prime}$ bands being only present at the root of the TGCs [12]. Meanwhile, the 
TGCs are of preferential zigzag monochirality, in which all constituent rolled graphene sheets have identical zigzag chirality $[11,15]$. In this kind of unique atomic configuration, it is possible that the TGCs have extraordinary electronic and phonon density of states compared with poly-chiral MWCNTs [30]. The van der Waals interaction between the constituent inner tubes in the TGCs is no longer akin to that in SWCNT bundles and general MWCNTs [31]. A strong resonant excitation may occur in these TGCs, similar to that observed in SWCNTs directly or MWCNTs with the magnification normally associated with electromagnetic and chemical enhancement effects [3, 10]. This is supported by the poor depolarization effect from the angular independent intensity behaviour. As a result, the split $\mathrm{G}$ band can be found directly from highly crystalline and monochiral TGCs that are only $20 \%$ in the sample, without any external enhancement effects. The G band splitting can't be accounted for solely by other factors (such as the stress, small-size and curvature effects etc.) than the structural monochirality, because the as-deposited TGC is a straight, rigid and isolated nanostructure freely standing on the carbon spherules and no structural deformation can happen inside. Thus, in terms of a unique microstructure of TGCs, its characteristic Raman spectra presented here may be used as a fingerprint of monochirality of tubular carbon-based materials. The detailed corresponding relation between the peak frequency and the structure of TGCs needs to be further investigated by both theoretical and experimental studies.

\section{Conclusion}


In summary, we report a low-frequency peak at $280 \mathrm{~cm}^{-1}$ and a split $\mathrm{G}$ band at $1569-$ $1587 \mathrm{~cm}^{-1}$, directly from as-deposited individual TGCs without the help of any surface electromagnetic or chemical enhancement effects. The low-frequency peak originates from either the RBM of the innermost nanotube in the TGC or iTA mode of graphite. The split $\mathrm{G}$ band can be fitted with two Lorentzian peaks at $1571 \pm 2$ and $1584 \pm 3 \mathrm{~cm}^{-1}$, which are ascribed to atomic vibrations along the circumference of individual innermost tube and collective atomic vibrations along the axis of constituent all carbon tubes of TGCs, respectively, due to what we believe are monochirality enhanced resonance excitation which is supported by the study of the polarized Raman G band. The present Raman study shows that the as-deposited TGC is of high crystalline quality and the G band splitting may be considered as a fingerprint of the monochirality of tubular carbon nanomaterials. Both the highly crystalline quality and the monochirality of TGCs play a major role in the finding of the low-frequency mode and structured $\mathrm{G}$ band.

\section{Acknowledgments}

Authors thank V. Scardaci in the Engineering Department of Cambridge University for help with Raman measurements and J. D. Carey in the Advanced Technology Institute, University of Surrey for his kind discussion. This work was financially supported by the Engineering and Physical Science Research Council (EPSRC) Portfolio Partnership Award under Grant GR/S975901, UK, the Ministry for Education and Research (BMBF) under Project CHN 05/002 of WTZ, Germany, and the European Union under the DESYGN-IT project (STREP Project 505626-1). 


\section{Reference:}

[1] Ferrari AC, Meyer JC, Scardaci V, Casiraghi C, Lazzeri M, Mauri F, et al. Raman spectrum of graphene and graphene layers. Phys. Rev. Lett. 2006;97:187401-187404.

[2] Reich S, Thomsen C. Raman spectroscopy of graphite. Phil. Trans. R. Soc. Lond. A 2004;362:2271-2288.

[3] Dresselhaus MS, Dresselhaus G, Jorio A, Filho AGS, Saito R. Raman spectroscopy on isolated single wall carbon nanotubes. Carbon 2002;40:2043-2061.

[4] Prawer S, Nemanich RJ. Raman spectroscopy of diamond and doped diamond. Phil. Trans. R. Soc. Lond. A 2004;362:2537-2565.

[5] Silva SRP, Amaratunga, GAJ, Salje EKH. Evidence of hexagonal diamond in plasmadeposited carbon films J. Mater. Sci. 1994;29: 4962-4966.

[6] Kuzmany H, Pfeiffer R, Hulman M, Kramberger C. Raman spectroscopy of fullerenes and fullerene-nanotube composites. Phil. Trans. R. Soc. Lond. A 2004;362:2375-2406.

[7] Schwan J, Ulrich S, Batori V, Ehrhardt H, Silva SRP. Raman spectroscopy on amorphous carbon films. J. Appl. Phys. 1996;80:440-447.

[8] Silva SRP, Rafferty B, Amaratunga GAJ, Schwan J, Franceschini DF, Brown LM. Nitrogenated amorphous carbon as a semiconductor. Diamond and Related Mater. 1996;5:401-404.

[9] Zhou O, Fleming RM, Murphy DW, Chen CH, Haddon RC, Ramirez AP, et al. Defects in carbon nanostructures. Science 1994;263:1744-1747. 
[10] Zhao XL, Ando Y, Qin L, Kataura H, Maniwa Y, Saito R. Multiple splitting of Gband modes from individual multiwalled carbon nanotubes. Appl. Phys. Lett. 2002;81:2550-2552.

[11] Zhang GY, Jiang X, Wang EG. Tubular graphite cones. Science 2003;300:472-474.

[12] Shang NG, Milne WI, Jiang X. Tubular graphite cones with single-crystal nanotips and their antioxygenic properties. J. Am. Chem. Soc. 2007;129:8907-8911.

[13] Zhang YY, Zhang J, Son HB, Kong J, Liu ZF. Substrate-induced Raman frequency variation for single-walled carbon nanotubes. J. Am. Chem. Soc. 2005;127:17156-17157.

[14] Duan XJ, Son HB, Gao B, Zhang J, Wu TJ, Samsonidze GG, et al. Resonant raman spectroscopy of individual strained single-wall carbon nanotubes. Nano Lett. 2007;7:2116-2121.

[15] Zhang GY, Bai XD, Wang EG, Guo Y, Guo WL. Monochiral tubular graphite cones formed by radial layer-by-layer growth. Phys. Rev. B 2005;71:113411/1-4.

[16] Saito R, Jorio A, Filho AGS, Grueneis A, Pimenta MA, Dresselhaus G, et al. Dispersive Raman spectra observed in graphite and single wall carbon nanotubes. Physica B 2002;323:100-106.

[17] Tan PH, Hu CY, Dong J, Shen WC, Zhang BF. Polarization properties, high-order Raman spectra, and frequency asymmetry between Stokes and anti-Stokes scattering of Raman modes in a graphite whisker. Phys. Rev. B 2001;64:214301/1-12.

[18] Jorio A, Filho AGS, Dresselhaus G, Dresselhaus MS, Swan AK, Ünlü MS, et al. Gband resonant Raman study of 62 isolated single-wall carbon nanotubes. Phys. Rev. B 2002;65:155412/1-9. 
[19] Saito R, Jorio A, Hafner JH, Lieber CM, Hunter M, McClure T, et al. Chiralitydependent G-band Raman intensity of carbon nanotubes. Phys. Rev. B 2001;64:085312/1-7.

[20] Duesberg GS, Loa, I, Murghard, Syassen K, Roth S. Polartized Raman spectroscopy on isolated single-wall carbon nanotubes. Phys. Rev. Lett. 2000;85:5436-5439.

[21] Rao AM, Jorio A, Pimenta MA, Dantas MSS, Saito R, Dresselhaus, G, Dresselhaus MS. Polarized Raman study of aligned multiwalled carbonnanotubes. Phys. Rev. Lett. 2000;84:1820-1823.

[22] Jorio A, Filho AGS, Brar VW, Swan AK, Ünlü MS, Goldberg BB, et al. Polarized resonant Raman study of isolated single-wall carbon nanotubes: symmetry selection rules, dipolar and multipolar antenna effects. Phys. Rev. B 2002;65:121402/1-4.

[23] Chen G, Wu J, Lu Q, Gutierrez HR, Xiong Q, Pellen, ME, et al. Optical antenna effect in semiconducting nanowires. Nano Lett. 2008;8:1341-1346.

[24] Fantini C, Pimenta MA, Dantas MSS, Ugarte D, Rao AM, Jorio A, et al. MicroRaman investigation of aligned single-wall carbon nanotubes. Phys. Rev. B $2001 ; 63: 161405 / 1-4$.

[25] Kwon YK, Tománek D. Electronic and structural properties of multiwall carbon nanotubes. Phys. Rev. B 1998;58:R16001-R16004.

[26] Ho GW, Ho YH, Li TS, Chang, CP, Lin MF. Band structure and absorption spectrum of double walled zigzag carbon nanotubes in an electric field. Carbon 2006: 44:2323-2329.

[27] Jantoljak H, Salvetat JP, Forró L, Thomsen C. Low-energy Raman-active phonons of multiwalled carbon nanotubes. Appl. Phys. A 1998;67:113-116. 
[28] Benoit JM, Buisson JP, Chauvet O, Godon C, Lefrant S. Low-frequency Raman studies of multiwalled carbon nanotubes: Experiments and theory. Phys. Rev. B. 2002;66:73417/1-4.

[29] Zhao XL, Ando Y, Qin LC, Kataura H, Maniwa Y, R. Saito. Radial breathing modes of multiwalled carbon nanotubes. Chem. Phys. Lett. 2002;361:169-174.

[30] Kahn D, Lu JP. Vibrational modes of carbon nanotubes and nanoropes. Phys. Rev. B 1999;60:6535-6540.

[31] Rao AM, Chen J, Richter E, Schlecht U, Eklund PC, Haddon RC, et al. Effect of van der Waals interactions on the Raman modes in single walled carbon nanotubes. Phys. Rev. Lett. 2001;86:3895-3898. 